\title{
RNA interference for epidermal growth factor receptor enhances the radiosensitivity of esophageal squamous cell carcinoma cell line Eca109
}

\author{
HEPING ZHANG ${ }^{1 *},{\text { JIANCHENG } \text { LI }^{2 *}, \text { WENFANG CHENG }}^{2}$, DI LIU ${ }^{2}, \mathrm{CHENG} \mathrm{CHEN}^{2}$, \\ XIAOYING WANG ${ }^{2}$, XUJING LU $^{1}$ and XIFA ZHOU ${ }^{1}$ \\ ${ }^{1}$ Department of Radiation Oncology, Changzhou Tumor Hospital of Soochow University, Changzhou, \\ Jiangsu 213002; ${ }^{2}$ Department of Radiation Oncology, Fujian Provincial Tumor Hospital, \\ Provincial Clinical College of Fujian Medical University, Fuzhou, Fujian 350014, P.R. China
}

Received July 7, 2014; Accepted April 13, 2015

DOI: $10.3892 / \mathrm{ol} .2015 .3456$

\begin{abstract}
The present study investigated the effects of small interfering RNAs (siRNAs) specific to the epidermal growth factor receptor (EGFR) gene, on the radiosensitivity of esophageal squamous cell carcinoma cells. EGFR gene siRNAs (EGFR-siRNA) were introduced into esophageal cancer Eca109 cells using Lipofectamine ${ }^{\circledR} 2000$. The EGFR messenger (m)RNA expression levels, EGFR protein expression and cell growth were assessed using reverse transcription-polymerase chain reaction analysis, western blot analysis and a Cell Counting Kit-8 (CCK-8), respectively. In addition, colony assays were used to determine the inhibitory effects of $\mathrm{X}$-ray radiation on EGFR-silenced cells. EGFR mRNA and protein levels were reduced in the Eca109 cells transfected with EGFR-siRNA. The relative EGFR mRNA expression levels were reduced to 26.74, 9.52 and $4.61 \%$ in Eca109 cells transfected with EGFR-siRNA1, 2 and 3, respectively. These mRNA levels were significantly reduced compared with the those of the control group (42.44\%; $\mathrm{P}<0.0001)$. Transfection with siRNA3 resulted in the greatest reduction in EGFR mRNA expression, with an inhibition rate of $85 \%$. The relative EGFR protein expression levels were reduced to 24.05, 34.91 and $34.14 \%$ in Eca109 cells transfected with EGFR-siRNA1, 2 and 3, respectively. These protein levels were significantly reduced compared with those of the control group (78.57\%;
\end{abstract}

Correspondence to: Dr Xujing Lu or Dr Xifa Zhou, Department of Radiation Oncology, Changzhou Tumor Hospital of Soochow University, 1 Huaide North Road, Changzhou, Jiangsu 213002, P.R. China

E-mail: luxujing68@aliyun.com

E-mail: zhouxifacz@sina.com

*Contributed equally

Key words: small interfering RNA, radiosensitivity, epidermal growth factor receptor, cell lines, esophageal neoplasm
$\mathrm{P}<0.0001)$. Transfection with siRNA1 resulted in the greatest reduction in EGFR protein expression, with an inhibition rate of $72.84 \%$. This reduction in EGFR expression inhibited the proliferation of Eca109 cells, which was identified using the CCK- 8 assay. The proliferation inhibition ratio was $28.2 \%$. The cells treated with irradiation in addition to EGFR-siRNA, demonstrated reduced radiobiological parameters (D0, Dq and SF2) compared with those of cells treated with irradiation only, with a sensitization enhancing ratio of 1.5. In conclusion, suppression of EGFR expression may enhance the radiosensitivity of esophageal cancer Eca109 cells and therefore may represent a promising approach for future clinical practice.

\section{Introduction}

Esophageal squamous cell carcinoma (ESCC) is one of the most common digestive tract cancers, and accounts for approximately one-sixth of all cancer-associated mortalities worldwide. There are $\sim 300,000$ cases of esophageal cancer (EC)-associated mortalities annually worldwide (1). The incidence of ESCC is characterized by distinctive geographic distribution, with half of all cases worldwide occurring in China. Approximately $70 \%$ of patients present with advanced ESCC at the time of diagnosis and treatment consists of radiotherapy and chemotherapy, in addition to palliative care for terminally ill patients. The prognosis for EC is poor, with a 5 -year survival rate of $\leq 20 \%$ (2) and therefore, ESCC continues to result in high mortality rates. Although various refinements of the conventional therapies for EC, including surgery, radiotherapy, chemotherapy and their multidisciplinary applications have been attempted, improvements in the overall survival rate remain unsatisfactory (3). Therefore, the development of novel therapies to treat $\mathrm{EC}$ are urgently required.

The epidermal growth factor receptor (EGFR) is involved in multiple signaling pathways, a number of which are associated with esophageal cancer progression. EGFR is a major regulator in a variety of physiological processes, including cell growth, differentiation, apoptosis and cell death (4). In the development of tolerance to irradiation, an intracellular phosphorylation cascade initiated by auto-phosphorylation 
of transmembrane tyrosine kinase receptors, and of EGFR (also known as HER-1) in particular, has been recognized as a signaling pathway associated with cell survival (5). The high expression and mutation rate of EGFR may result in radiotherapy resistance, leading to a reduction in radiosensitivity. EGFR is currently being studied as a potential ESCC therapeutic target (6).

RNA interference (RNAi) is a highly specific method of gene silencing that utilizes small interfering RNA (siRNA) molecules complementary to the target sequence (7). These siRNAs bind to gene transcripts, targeting them for early destruction prior to translation (7). RNAi techniques are already extensively used in research but also have promising potential for the development of drugs capable of targeting specific proteins $(8,9)$, including EGFR (10). In the present study, siRNA technology was used to reduce EGFR expression in ESCC cells, and its effects on cell proliferation and radiosensitivity were assessed. The results of the present study may provide the theoretical basis on which to develop novel treatments for ESCC.

\section{Materials and methods}

Cell culture and major reagents. Human ESCC Eca109 cells were purchased from the Shanghai Institute of Cell Biology (Shanghai, China). The cells were cultured in RPMI-1640 supplemented with $10 \%$ fetal calf serum (Gibco Life Technologies, Carlsbad, CA, USA) and maintained at $37^{\circ} \mathrm{C}$ under a humidified atmosphere of $5 \% \mathrm{CO}_{2}$. TRIzol reagent, liposomes and Lipofectamine 2000 were purchased from Invitrogen Life Technologies (Carlsbad, CA, USA). The RevertAid First Strand cDNA Synthesis Kit was purchased from Thermo Fisher Scientific, Inc. (Pittsburgh, PA, USA) and the Fast HiFidelity PCR kit was purchased from Tiangen Biotech Co., Ltd. (Beijing, China). The EGFR primer sequences were designed and synthesized by Invitrogen Life Technologies, the EGFR primary antibody was purchased from Abcam (Cambridge, UK), the secondary antibody and the Micro Bicinchoninic Acid (BCA) Protein Assay Kit were purchased from Thermo Fisher Scientific, Inc. (Waltham, MA, USA), and the radioimmunoprecipitation assay (RIPA) protein lysis buffer and Cell Counting Kit-8 (CCK-8) kit were purchased from Beyotime Institute of Biotechnology (Guangzhou, China).

Design and synthesis of siRNAs. A total of three siRNA pairs targeting the EGFR gene were designed and synthesized by Invitrogen Life Technologies. The sequences used were as follows: EGFR-siRNA1 sense, UGAUCUGUCACCACAUAA UUACGG and antisense, CCCGUAAUUAUGUGGUGACAG AUCA; EGFR-siRNA2 sense, UUAGAUAAGACUGCUAAG GCAUAGG and antisense, CCUAUGCCUUAGCAGUCU UAUCUAA; EGFR-siRNA3 sense, UUUAAAUUCACCAAU ACCUAUUCCG and antisense, CGGAAUAGGUAUUGG UGAAUUUAAA.

Cell transfection. The cells were divided into 4 groups: The EGFR-siRNA-treated group, the positive siRNA control group, the non-targeting siRNA-treated group (negative control) and the untreated control group (blank). Eca109 cells were seeded into six-well plates at a density of $5 \times 10^{5}$ cells/well. When the cells reached $80-90 \%$ confluency, negative control siRNA (Guangzhou RiboBio Co., Ltd., Guangzhou, China) or EGFRsiRNA (final concentration, $50 \mathrm{nmol} / \mathrm{l}$; Guangzhou RiboBio Co., Ltd.) was transfected according to the Lipofectamine 2000 transfection instructions. The transfection efficiency was determined using fluorescently labeled siRNA and an Olympus IX70 Inverted Fluorescent Microscope (Olympus Corporation, Tokyo, Japan). The cells were irradiated prior to harvest for RNA and protein measurements, following transfection for $48 \mathrm{~h}$.

Reverse transcription-polymerase chain reaction (RT-PCR) to detect EGFR mRNA expression. The total RNA was extracted using TRIzol reagent and then reverse transcribed into cDNA using the RevertAid First Strand cDNA Synthesis Kit. $\beta$-actin was selected as an internal reference gene (1). The EGFR primers (Invitrogen Life Technologies) were as follows: Forward (F), 5'-AAAGACCTGTACGCCAAC ACAG-3'; and reverse (R), 5'-TTTTAGGATGGCAAGGGA CTTC-3', which amplified a fragment of 244 base pairs (bp). The cycling conditions consisted of $94^{\circ} \mathrm{C}$ pre-incubation for $4 \mathrm{~min}$, followed by 30 cycles of denaturation at $94^{\circ} \mathrm{C}$ for $30 \mathrm{sec}$, annealing at $60^{\circ} \mathrm{C}$ for $30 \mathrm{sec}$ and extension at $72^{\circ} \mathrm{C}$ for $1 \mathrm{~min}$, followed by a final extension at $72^{\circ} \mathrm{C}$ for $10 \mathrm{~min}(2)$. The internal reference ( $\beta$-actin) gene primers were $\mathrm{F} 5^{\prime}$-AAA GACCTGTACGCCAACACAG-3' and R 5'-TTTTAGGAT GGCAAGGGACTTC-3', which amplified a fragment of $556 \mathrm{bp}$. Equal volumes of the PCR products were subjected to electrophoresis on $2 \%$ agarose gels and fragments were visualized by ethidium bromide staining. The grayscale images of the PCR amplification products were analyzed using a gel imaging system (ChemiDoc XRS+ system) and Quantity One 1-D Analysis software (Bio-Rad Laboratories, Inc., Hercules, CA, USA).

CCK-8 assay. Eca109 cells were seeded in 96-well plates at a density of $6 \times 10^{3}$ cells/well. All experiments were repeated three times, and the mean results were recorded. The culture plates were incubated at $37^{\circ} \mathrm{C}$ with $5 \% \mathrm{CO}_{2}$ in a humidified air incubator. Following treatment with EGFR-siRNA3, the cells were cultured for a further $24 \mathrm{~h}$, prior to the addition of $10 \mu \mathrm{l}$ CCK-8 solution $(10 \mu \mathrm{g} / \mathrm{l})$ to every well in the dark. The cells were incubated for a further $2 \mathrm{~h}$. ELISA was then performed and the absorbance of each well was measured at $490 \mathrm{~nm}$ (OD, optical density). The cell proliferation inhibition rate was calculated using the following formula: Cell proliferation inhibition rate $(\%)=[(1$-treated group OD value $) /$ control group OD value] x $100 \%$.

Western blot analysis. The total protein was extracted from the Eca109 cells using RIPA lysis buffer and the protein concentration was determined using a Micro BCA Protein Assay Kit. The cell lysates were centrifuged at 8,000 x g for $5 \mathrm{~min}$. The total proteins $(50 \mu \mathrm{g})$ were separated by $10 \%$ SDS-PAGE (Invitrogen Life Technologies) and transferred to a nitrocellulose membrane (Yubo Biology Co., Ltd., Shanghai, China). The membrane was blocked for $1 \mathrm{~h}$ at room temperature in phosphate-buffered saline with Tween-20 (PBST; $0.05 \%$ Tween-20; Thermo Fisher Scientific, Inc.) containing 
$5 \%$ skimmed milk. The membranes were washed three times with PBST, and then incubated overnight at $4^{\circ} \mathrm{C}$ with the primary monoclonal rabbit anti-human $\operatorname{EGFR~}(1: 1,000$; cat. no. ab76153; Abcam) and rabbit anti-human $\beta$-tubulin (1:500; cat. no. ab179513; Abcam) antibodies diluted in PBST. Following three washes with PBST, the membranes were incubated with polyclonal goat anti-rabbit secondary antibody (1:500; cat. no. 32260; Thermo Fisher Scientific, Inc.) for $1 \mathrm{~h}$ at room temperature. The membranes were washed 4 times with PBST, and detection was performed using 3,3'-diaminobenzidine (Pierce DAB Substrate Kit; Thermo Fisher Scientific, Inc.). The band intensity was digitized and analyzed using Quantity One 1-D software.

Irradiation and clonogenic assay. Cells ( 300$)$ that had been treated with EGFR-siRNA3 or a non-targeting siRNA as the control were seeded into 60-mm diameter culture dishes. Each group was irradiated at six different doses: 0, 1, 2, 4, 6 and $8 \mathrm{~Gy}$, using the 6MV-X Synergy linear accelerator (Elekta, Stockholm, Sweden) with a 1-cm filter and a source-skin distance of $100 \mathrm{~cm}$, at a dose rate of $3 \mathrm{~Gy} / \mathrm{min}$. The radiation field was $40 \times 40 \mathrm{~cm}$. Following 10 days of incubation, the colonies were fixed with methanol and stained with Giemsa (Beijing Dingguo Changsheng Biotechnology Co., Ltd., Beijing, China) for $30 \mathrm{~min}$. Colonies consisting of $\geq 50$ cells were scored as viable colonies. The colony formation rate was estimated from the planting efficiency (PE; number of clones/inoculated cells $\times 100 \%$ ) and the surviving fraction (SF; colony formation rate of irradiated cells/colony formation rate of control cells x $100 \%$ ). The data were fit to the multi-target model, and a survival curve of each group was established using Prism software, version 5.0 (GraphPad Software Inc., La Jolla, CA, USA). The radiobiological parameters, including D0, Dq, and SF2 (survival fraction at 2 Gy) were calculated according to the survival curves, in which $\log \mathrm{N}=\mathrm{Dq} / \mathrm{D} 0$. The sensitization enhancement ratio was calculated as the SF2 of the control group/SF2 of the EGFR-siRNA3-treated group. The results are based on $\geq 3$ independent experiments.

Statistical analysis. The data are expressed as the mean \pm standard error of the mean, and the one-way analysis of variance (ANOVA) was performed using SPSS software version 17.0 (SPSS, Inc., Chicago, IL, USA), in addition to the Least Significant Difference test. A P-value of $<0.05$ was considered to indicate a statistically significant difference.

\section{Results}

Eca109 cells are successfully transfected with siRNA. The siRNA was successfully transfected into Eca109 cells using Lipofectamine 2000, in a transient manner. Based on the expression of the red fluorescent $\mathrm{Cy} 3$ reporter (Fig. 1), it was demonstrated that EGFR-siRNA at $50 \mathrm{nmol} / 1$ yielded the highest transfection efficiency $(\leq 85 \%)$, and was therefore adopted for all subsequent experiments.

EGFR-siRNA treatment reduces EGFR mRNA expression. The EGFR mRNA relative expression levels were $26.74 \pm 3.40$, $9.52 \pm 1.09$ and $4.61 \pm 10.5 \%$ following transfection with EGFR siRNA1, 2 and 3, respectively; EGFR mRNA expression was
A

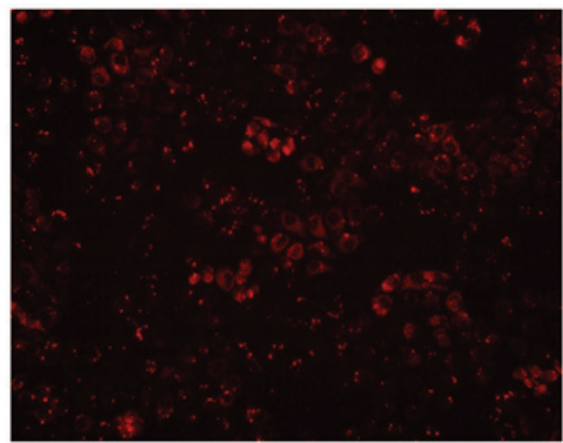

B

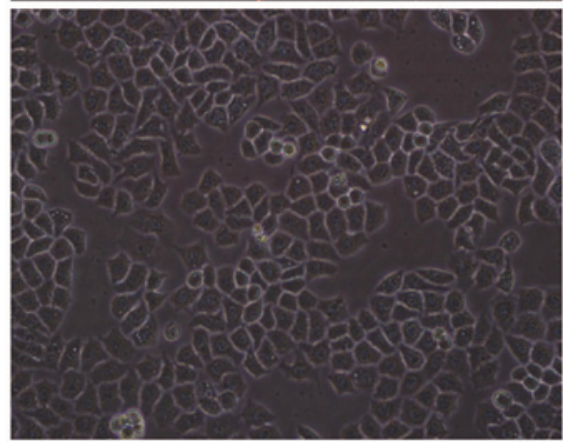

Figure 1. Eca109 cells successfully transfected with small interfering RNA 3. (A) Fluorescent imaging of $\mathrm{Cy} 3$ reporter (red) post-transfection, 200 bright fields. (B) Fluorescent microscope image post-transfection, 200 dark fields.

A

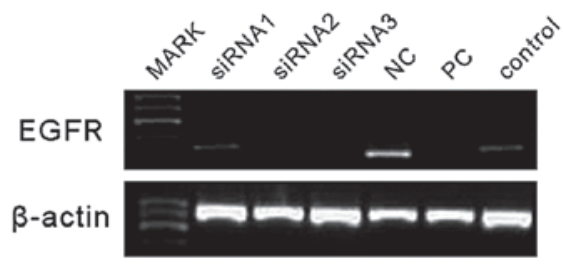

B

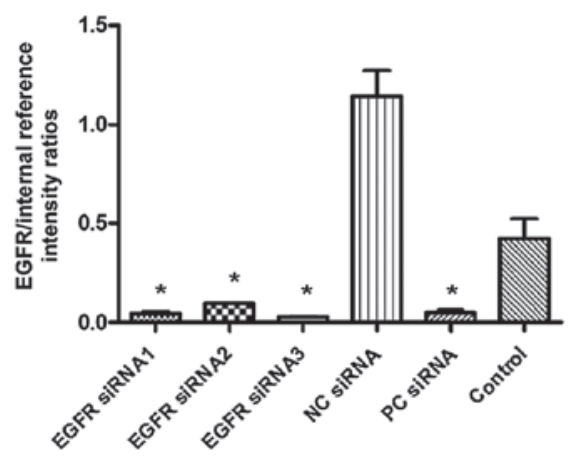

Figure 2. EGFR-siRNA transfection reduces EGFR mRNA expression levels. (A) Reverse transcription-PCR was used to detect the EGFR and $\beta$-actin gene expression in the various groups $48 \mathrm{~h}$ following transfection. (B) PCR products were semi-quantified by assessing the relative intensity of messenger RNA expression using image analysis by comparing EGFR with $\beta$-actin. Data are presented as the mean \pm standard deviation of three independent experiments ( $\mathrm{P}<0.0001$ vs. Control). EGFR, epidermal growth factor receptor; siRNA, small interfering RNA; PCR, polymerase chain reaction; NC, non-targeting siRNA (negative control); PC, postitive interference siRNA (positive control); Control, blank control group (untransfected).

significantly reduced, compared with the relative expression rate of $42.44 \%$ in the control group $(\mathrm{P}<0.0001$; Fig. 2$)$. The inhibition efficiency of the three EGFR-siRNAs on EGFR mRNA expression was 22,70 and $85 \%$, respectively. As the 
Table I. OD values for Eca109 cell proliferation following transfection with EGFR-siRNA3 ( $\mathrm{n}=3$ ).

\begin{tabular}{ll}
\hline Group & OD value \\
\hline Blank control group & $1.847 \pm 0.641$ \\
Positive interference siRNA control & $1.499 \pm 0.2974^{\mathrm{a}}$ \\
Negative interference siRNA control & $1.820 \pm 0.548$ \\
EGFR-siRNA & $1.326 \pm 0.110^{\mathrm{a}}$ \\
\hline
\end{tabular}

Values are expressed as the mean \pm standard error of the mean. ${ }^{\mathrm{a}} \mathrm{P}<0.05$ vs. blank control group. OD, optical density; EGFR, epidermal growth factor receptor; siRNA, small interfering RNA.

Table II. Radiobiological parameters fitted using the multi-target model.

\begin{tabular}{lcccc}
\hline Group & D0 & Dq & SF2 & SER \\
\hline Blank control & 2.86 & 0.88 & 0.48 & \\
EGFR-siRNA & 1.84 & 0.65 & 0.32 & 1.5 \\
\hline
\end{tabular}

SF, survival fraction at $2 \mathrm{~Gy}$; D0, mean lethal dose, also used as the final slope; Dq, quasi-threshold dose; SER, sensitization enhancement ratio; EGFR, epidermal growth factor receptor; siRNA, small interfering RNA.

A

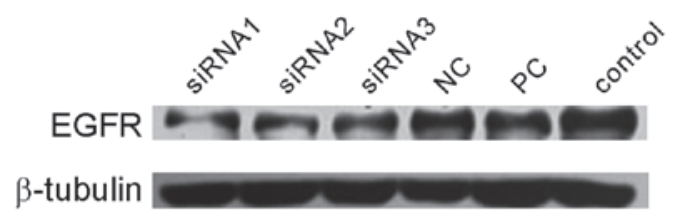

B

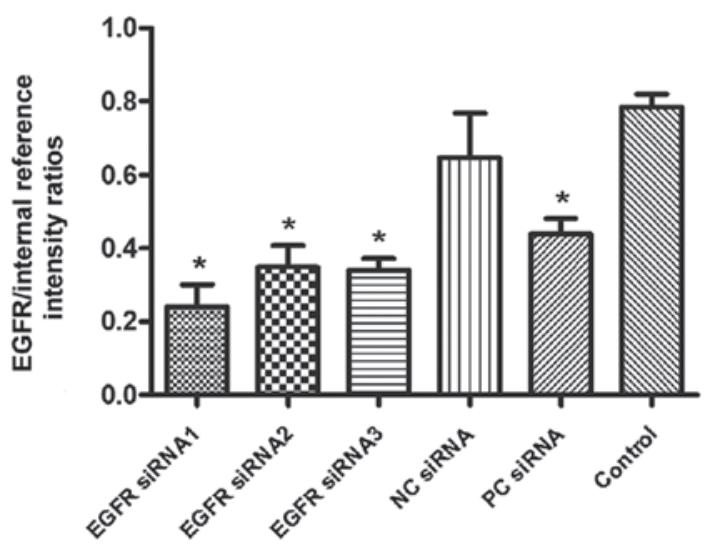

Figure 3.EGFR-siRNA treatment reduces EGFR protein expression. (A) EGFR and $\beta$-tubulin protein expression in the various groups was detected by western blotting. (B) Semi-quantitative analysis of the EGFR protein expression comparing EGFR to $\beta$-tubulin. Data are presented as the mean \pm standard deviation of three experiments; "P $<0.0001$ vs. Control). NC, negative interference siRNA; PC, positive interference siRNA; Control, blank control group (untransfected); EGFR, epidermal growth factor receptor; siRNA, small interfering RNA.

EGFR mRNA levels were most significantly reduced in the EGFR-siRNA3 group, the EGFR-siRNA3 was selected for use in the subsequent proliferation and clonogenic assays.

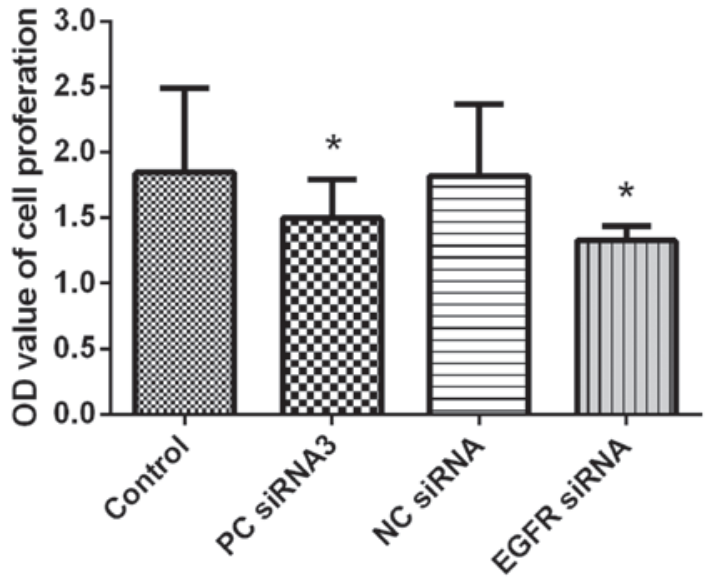

Figure 4. EGFR-siRNA treatment inhibits Eca109 cell proliferation asdetermined by CCK- 8 assay. OD values of Eca109 cell proliferation following transfection with EGFR-siRNA. Results are presented as mean \pm standard deviation of four separate experiments (" $\mathrm{P}<0.05$ vs. Control). NC, negative interference siRNA; PC, positive interference siRNA; Control, blank control group (untransfected); EGFR, epidermal growth factor receptor; siRNA, small interfering RNA; OD, optical density.

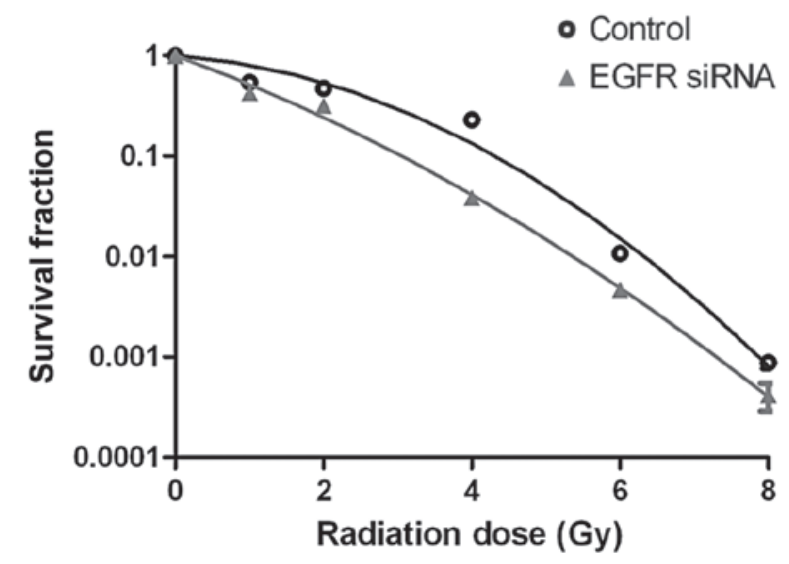

Figure 5. EGFR-siRNA increases the radiosensitivity of Eca109 cells. Survival curve of EGFR-siRNA and control Eca109 cells treated with various doses of X-ray irradiation. EGFR, epidermal growth factor receptor; siRNA, small interfering RNA.

EGFR-siRNA treatment reduces EGFR protein expression. The EGFR protein relative expression rate was $24.05 \pm 6.01$, $34.91 \pm 5.78$ and $34.14 \pm 3.08 \%$ (Fig. 3) following transfection with EGFR-siRNA1, 2 and 3, respectively, compared with $78.57 \%$ in the control group. This reduction in EGFR protein level was significant $(\mathrm{P}<0.0001)$. The inhibition efficiencies of the three types of EGFR-siRNA on EGFR protein expression were $72.84,53.01$ and $56.21 \%$ (Fig. 3).

Downregulation of EGFR expression inhibits the growth of Ecal09 cells. EGFR-siRNA-treated cells demonstrated notable growth retardation at $48 \mathrm{~h}$ following transfection, with an inhibition rate of $28.2 \%$, compared with the rate of growth in the blank control group (Fig. 4; Table I). This inhibition rate was greater than that observed at $24 \mathrm{~h}$ (data not shown), and therefore, this time-period was selected as the detection time for mRNA and protein expression of EGFR. 
EGFR-siRNA increases the radiosensitivity of Ecal09 cells. The survival curve for the Eca109 cells in the EGFR-siRNA group was significantly reduced following exposure to various doses of X-ray radiation, compared with that of the control group (Fig. 5). The radiobiological parameters are presented in Table II, with notable differences between the D0 and Dq values of the two groups. These results indicated that transfection with EGFR-siRNA reduced the ability of the Eca109 cells to repair X-ray-induced damage, and therefore, increases the radiosensitivity of the Eca109 cells.

\section{Discussion}

Binding of a ligand to the extracellular domain of the EGFR results in dimerization of the EGFR molecules in the cell membrane and induction of intracellular tyrosine kinase activity. Autophosphorylation of specific tyrosine residues in the tyrosine kinase domain results in a series of intracellular signal cascade amplifications and transmissions, which ultimately regulate nuclear gene expression resulting in cell proliferation and differentiation (11). Previous studies have demonstrated that EGFR is involved in the development and progression of numerous types of tumor, thus making it an important target for anticancer drug design (12). In the present study, the role of EGFR was examined in the Eca109 ESCC cell line, in which EGFR is highly expressed.

Overexpression or mutation of EGFR is one of the main mechanisms underlying tumor cell resistance to radiotherapy, which results in poor prognosis (13). Akimoto et al (5) demonstrated that the expression of EGFR and radiosensitivity of solid tumors was negatively correlated, in in vitro experiments. Tumors expressing high levels of EGFR were more resistant to radiation compared with tumors expressing low levels of EGFR (13). Therefore, EGFR may represent an effective target for radiosensitization. The EGFR inhibitor cetuximab (IMC-c225) and EGFR tyrosine kinase inhibitors, including gefitinib and erlotinib, have previously been comprehensively investigated (14), and a proportion of these drugs have also been tested in phase I to phase III clinical trials for the treatment of ESCC (14-18). However, as these drugs are only effective in a small number of patients, novel methods of inhibiting the EGFR pathway are required.

RNAi is a mechanism of post-transcriptional gene silencing, which has been observed in a number of eukaryotes. siRNAs targeting specific genes in tumor cells may be used to inhibit tumor cell growth, induce apoptosis and enhance sensitivity to radiotherapy and chemotherapy; thus, they possess significant potential as novel cancer drugs (19). Zhang et al (20) synthesized a combined double-stranded RNA with a specific EGFR sequence by chemical methods in vitro, transfected it into A549 cells with Lipofectamine 2000 and observed that EGFR expression was reduced by $>70 \%$, as measured by western blot analysis and flow cytometry, while the cell growth inhibition rate was $>70 \%$. Huo et al (21) transfected Eca109 cells with an extracellular signal regulated kinase-specific siRNA to inhibit the mitogen-activated protein kinase signaling pathway. This pathway functions downstream of EGFR signaling. The authors demonstrated that interference with this pathway inhibited tumor cell growth, induced apoptosis, arrested cell cycle progres- sion and enhanced the radiosensitivity of EC cells. In the present study, EGFR expression was successfully reduced in ESCC Eca109 cells by transfection with EGFR-siRNA using Lipofectamine 2000. Following transfection with EGFR-siRNA, the inhibition rate of EGFR mRNA was as high as $85 \%$ and EGFR protein inhibition was as high as $72.84 \%$, following EGFR knockdown in Eca109 cells. In addition, the CCK-8 assay demonstrated that Eca109 cell growth was inhibited as a result of EGFR downregulation, with an inhibition rate of $28.2 \%$. These results indicated that the EGFR gene is important in the proliferation and health of ESCC cells. A previous study indicated that the use of RNAi technology can enhance the radiosensitivity of tumor cells (22). In the present study, a clonogenic assay was used to determine whether suppression of EGFR affected the radiosensitivity of Eca109 cells. The observed decline in the cell survival curve of EGFR-siRNA-treated cells compared with that of the control cells indicated that downregulation of EGFR increased cell radiosensitivity by up to 1.5 -fold. As RNAi technology continues to improve, it may be developed into a significant technique for the study of oncogene function.

In conclusion, the results of the present study indicated that RNAi technology successfully reduced EGFR mRNA and protein levels in ESCC cells, which subsequently resulted in inhibition of tumor cell proliferation in addition to an improvement in the sensitivity of tumor cells to radiation. These results provide a rationale for combining siRNA and radiotherapy in the treatment of human epithelial cancers, which express EGFR. Radiotherapy combined with siRNA may improve the therapeutic effect and reduce the total radiation dose required in a clinical setting, which is of potential clinical interest.

\section{Acknowledgements}

The present study was funded by the National Natural Science Foundation of China (grant nos. 81402518 and 81472920), the Jiangsu Provincial Special Program of Medical Science (no. BL2012046) and the Changzhou Scientific Program (nos. ZD201315 and CY20130017).

\section{References}

1. Parkin DM, Bray FI and Devesa SS: Cancer burden in the year 2000. The global picture. Eur J Cancer 37 (Suppl 8): S4-S66, 2001.

2. Stoner GD, Wang LS and Chen T: Chemoprevention of esophageal squamous cell carcinoma. Toxicol Appl Pharmacol 224: 337-349, 2007.

3. Tatematsu N, Ezoe Y, Tanaka E, Muto M, Sakai Y and Tsuboyama T: Impact of neoadjuvant chemotherapy on physical fitness, physical activity and health-related quality of life of patients with resectable esophageal cancer. Am J Clin Oncol 36: 53-56, 2013.

4. Ayyappan S, Prabhakar D and Sharma N: Epidermal growth factor receptor (EGFR)-targeted therapies in esophagogastric cancer. Anticancer Res 33: 4139-4155, 2013.

5. Akimoto T, Hunter NR, Buchmiller L, Mason K, Ang KK and Milas L: Inverse relationship between epidermal growth factor receptor expression and radiocurability of murine carcinomas. Clin Cancer Res 5: 2884-2890, 1999.

6. Lammering G: Molecular predictor and promising target: Will EGFR now become a star in radiotherapy? Radiother Oncol 74: 89-91, 2005.

7. Pai SI, Lin YY, Macaes B, Meneshian A, Hung CF and Wu TC: Prospects of RNA interference therapy for cancer. Gene Ther 13: 464-477, 2006 
8. Davis ME, Zuckerman JE, Choi CH, Seligson D, Tolcher A, Alabi CA, Yen Y, Heidel JD and Ribas A: Evidence of RNAi in humans from systemically administered siRNA via targeted nanoparticles. Nature 464: 1067-1070, 2010.

9. Dorsett Y and Tuschl T: siRNAs: Applications in functional genomics and potential as therapeutics. Nat Rev Drug Discov 3: 318-329, 2004

10. Nozawa H, Tadakuma T, Ono T, Sato M, Hiroi S, Masumoto K and Sato Y: Small interfering RNA targeting epidermal growth factor receptor enhances chemosensitivity to cisplatin 5-fluorouracil and docetaxel in head and neck squamous cell carcinoma. Cancer Sci 97: 1115-1124, 2006.

11. Rodemann HP, Dittmann K and Toulany M: Radiation-induced EGFR-signaling and control of DNA-damage repair. Int J Radiat Biol 83: 781-791, 2007.

12. Pal SK and Pegram M: Epidermal growth factor receptor and signal transduction: Potential targets for anti-cancer therapy. Anticancer Drugs 16: 483-494, 2005.

13. Krause M, Prager J, Zhou X, Yaromina A, Dörfler A, Eicheler W and Baumann M: EGFR-TK inhibition before radiotherapy reduces tumour volume but does not improve local control: Differential response of cancer stem cells and nontumourigenic cells? Radiother Oncol 83: 316-325, 2007.

14. Sunpaweravong P, Sunpaweravong S, Sangthawan D, et al: Combination of gefitinib, cisplatin and 5-FU chemotherapy and radiation therapy (RT) in newly-diagnosed patients with esophageal carcinoma. J Clin Oncol 25: 4605, 2007.

15. Safran H, Suntharalingam M, Dipetrillo T, et al: Cetuximab with concurrent chemoradiation for esophagogastric cancer: Assessment of toxicity. Int J Radiat Oncol Biol Phys 70: 391-395, 2008.
16. Ruhstaller T, Pless M, Dietrich D, et al: Cetuximab in combination with chemoradiotherapy before surgery in patients with resectable, locally advanced esophageal carcinoma: A prospective, multicenter phase 1B/II Trial (SAKK 75/06). J Clin Oncol 29: 626-631, 2011.

17. Suntharalingam M, Dipetrillo T, Akerman P, et al: Cetuximab, paclitaxel, carboplatin, and radiation for esophageal and gastric cancer. J Clin Oncol 24: 4029, 2006.

18. Tomblyn MB, Goldman BH, Thomas CR Jr, et al; SWOG GI Committee: Cetuximab plus cisplatin, irinotecan, and thoracic radiotherapy as definitive treatment for locally advanced, unresectable esophageal cancer: A phase-II study of the SWOG (S0414). J Thorac Oncol 7: 906-912, 2012.

19. Izquierdo M: Short interfering RNAs as a tool for cancer gene therapy. Cancer Gene Ther 12: 217-227, 2005.

20. Zhang M, Zhang X, Bai CX, Chen J and Wei MQ: Inhibition of epidermal growth factor receptor expression by RNA interference in A549 cells. Acta Pharmacol Sin 25: 61-67, 2004.

21. Huo Q, Zheng ST, Tuersun A, et al: shRNA interference for extracellular signal-regulated kinase 2 can inhibit the growth of esophageal cancer cell line Eca109. J Recept Signal Transduct Res 30: 170-177, 2010

22. Vandersickel V, Maneini M, Marras E, et al: Lentivirus-mediated RNA interference of Ku70 to enhance radiosensitivity of human mammary epithelial cells. Int J Rediat Biol 86: 114-124, 2010. 